\title{
ANOTHER FIXED POINT THEOREM FOR PLANE CONTINUA
}

\author{
CHARLES L. HAGOPIAN
}

\begin{abstract}
A continuum $M$ is said to be $\lambda$ connected if every two points of $M$ can be joined by a hereditarily decomposable subcontinuum of $M$. Here we prove that a bounded plane continuum that does not have infinitely many complementary domains is $\lambda$ connected if and only if its boundary does not contain an indecomposable continuum. It follows that every $\lambda$ connected bounded nonseparating subcontinuum of the plane has the fixed point property.
\end{abstract}

If a nondegenerate point set is both connected and closed it is called a continuum. A set $X$ is said to have the fixed point property if for each map $f: X \rightarrow X$ there is a point $x \in X$ such that $f(x)=x$. H. Bell proved [1] that every bounded nonseparating plane continuum that has a hereditarily decomposable boundary has the fixed point property (for a different proof see [3]). Recently the author proved [2] that every bounded nonseparating plane continuum that is arcwise connected has a hereditarily decomposable boundary and therefore has the fixed point property. In this note the author's theorem is extended to $\lambda$ connected bounded nonseparating plane continua.

THEOREM 1. Suppose M is a bounded continuum in the plane $S$ that does not have infinitely many complementary domains. Then $M$ is $\lambda$ connected if and only if $\mathrm{Bd} M$ (the boundary of $M$ ) does not contain an indecomposable continuum.

Proof. Suppose Bd $M$ does not contain an indecomposable continuum. Then $\mathrm{Bd} M$ is the union of a finite number of hereditarily decomposable continua. Let $B_{1}$ denote a component of $\mathrm{Bd} M ; B_{1}$ is hereditarily decomposable. Let $A_{1}$ be an arc in $S$ irreducible from $B_{1}$ to $\mathrm{Bd} M-B_{1}$. Since only the endpoints of $A_{1}$ belong to $\mathrm{Bd} M$ and they lie in the boundaries of different complementary domains of $M$, the arc $A_{1}$ is a subset of $M$. Continuing this process it is clear that the union of a finite number of arcs

Presented to the Society, April 24, 1971; received by the editors April 21, 1971.

AMS 1970 subject classifications. Primary 54H25, 57A05, 47H10, 55C20; Secondary 54F20, 54F15.

Key words and phrases. The fixed point property, arcwise connectedness, $\lambda$ connected continua, hereditarily decomposable boundary, nonseparating plane continua.

(C) American Mathematical Society 1972 
with Bd $M$ produces a hereditarily decomposable subcontinuum $H$ of $M$. Clearly any interior point of $M$ may be joined to $H$ by an arc in $M$. Hence $M$ is $\lambda$ connected.

Assume that $M$ is $\lambda$ connected. Suppose there exists an indecomposable continuum $I$ in Bd $M$. Let $q$ be a point of $M-I$. Let $\left\{U_{n}\right\}$ be the elements of a countable base for the topology on $S$ that intersect $I$. Since $M$ is $\lambda$ connected, for each point $p$ of $I$, there exists a subcontinuum $L$ of $M$ that contains $\{p, q\}$ and does not contain $I$. For each positive integer $n$, let $H_{n}$ be the set of all points of $I$ that can be joined with $q$ by a continuum in $M-U_{n}$. Note that $I=\bigcup_{n=1}^{\infty} H_{n}$. For some integer $j$, the closure of $H_{j}$ contains a nonempty open subset of $I$. Hence there exists a continuum in $M-U_{j}$ that contains a nonempty open subset of $I$. Since every subcontinuum of $M$ that contains a nonempty open subset of $I$ contains $I$ [2, Theorem 1], this is a contradiction. Therefore $\mathrm{Bd} M$ does not contain an indecomposable continuum.

THEOREM 2. Every bounded $\lambda$ connected nonseparating plane continuum has the fixed point property.

\section{BIBLIOGRAPHY}

1. H. Bell, On fixed point properties of plane continua, Trans. Amer. Math. Soc. 128 (1967), 539-548. MR 35 \#4888.

2. C. L. Hagopian, A fixed point theorem for plane continua, Bull. Amer. Math. Soc. 77 (1971), 351-354.

3. K. Sieklucki, On a class of plane acyclic continua with the fixed point property, Fund. Math. 63 (1968), 257-278. MR 39 \#2139.

Department of Mathematics, Sacramento State College, Sacramento, CaliFORNIA 95819

Current address: Department of Mathematics, Arizona State University, Tempe, Arizona 85281 